\title{
Electron-Hole Transport in Capacitively Coupled 1D Arrays of Small Tunnel Junctions
}

\author{
M. Matters,* J. J. Versluys, and J. E. Mooij \\ Department of Applied Physics and Delft Institute for Microelectronics and Submicron Technology (DIMES), \\ Delft University of Technology, P.O. Box 5046, 2600 GA Delft, The Netherlands
}

(Received 23 May 1996)

\begin{abstract}
We have measured the current-voltage characteristics of two capacitively coupled 1D arrays of small tunnel junctions, where the coupling capacitance is significantly larger than the junction capacitance. We voltage biased only one of the arrays, while the current was measured simultaneously in both arrays. We find that, at low bias voltages, the currents in the two arrays are comparable in magnitude but opposite in direction. The currents are carried by tunneling electron-hole pairs that are bound by the charging energy of the coupling capacitance. [S0031-9007(97)02813-5]
\end{abstract}

PACS numbers: 74.50.+r, 71.35.-y, 73.23.Hk

Several experiments have demonstrated that the Coulomb interaction of the electrons plays an important role in systems of small tunnel junctions. The significant charging energy prohibits electron tunneling below a certain threshold voltage. The charging energy reveals the discrete nature of the electron charge in these systems [13]. In 1D arrays of small tunnel junctions, the Coulomb interaction leads to transport of charge solitons through the array [4]. The soliton length depends on the ratio between the junction capacitance and the self-capacitance of the islands in between the junctions.

Theoretical [5,6] and experimental [7] work on systems of small tunnel junctions has shown that electron transport in the Coulomb blockade regime is possible by electron tunneling through one or more virtual states of higher energy. This macroscopic quantum tunneling (MQT) of the charge or cotunneling is possible even at zero temperature, where charge is transferred through more than one junction in one event. It has been shown [5] that the rate of cotunneling is proportional to $\left(R_{K} / R\right)^{M}$, where $R_{K}=h / 2 e^{2}$ is the resistance quantum, $R$ is the junction tunnel resistance, and $M$ is the number of junctions involved in the cotunneling event. Generally, cotunneling leads to quantum leakage of the current in single-electron tunneling devices. The quantum leakage forms a problem for devices aiming at metrological accuracy of the current $[8,9]$.

In this paper we demonstrate that the Coulomb interaction of the electrons leads to a current in a 1D array of small tunnel junctions without a voltage bias. This current flows if the array is capacitively coupled to a second, voltage-biased, array. The coupling capacitance must be significantly larger than the junction capacitance. In that case, Averin et al. predicted [10] that the transport through the coupled arrays at low bias voltages is governed by tunneling of electron-hole pairs, that are bound by the electrostatic energy of the coupling capacitance. The currents in both arrays are comparable in magnitude but opposite in direction.

We consider the circuit depicted in Fig. 1(a). At low voltages, single-electron tunneling is suppressed due to the Coulomb blockade. Instead, an electron in one array and a hole in the other can be transported through the system. Electron tunneling and hole tunneling (i.e., electron tunneling in the opposite direction) in different arrays form one cotunneling event.

If the coupling capacitance $C_{c}$ is much larger than the junction capacitance $C$, the electrostatic energy $\epsilon$ of the system is, after a single electron has tunneled from a lead into one of the arrays at zero bias voltage [10]:

$$
\epsilon=\frac{e^{2}}{4 C}\left(1-\frac{1}{N}\right),
$$

where $N$ is the number of junctions in one array. After a single-electron tunneling event, the energy can be lowered to $e^{2} / C_{c}$ by tunneling of an electron in the opposite direction in the other array. In this way, an electron-hole pair is created that can be called an exciton with a binding energy of the order of $\epsilon$. At voltages between $e / C_{c}$ and $e / 4 C$, electron-hole pairs can move through the system, giving rise to opposite currents in the arrays. At larger voltages $(e / 4 C<V<e / 2 C)$, the charge configuration created by the moving excitons can make single-electron tunneling energetically favorable. This leads to deviations from the simple exciton picture, in which both currents are equal in magnitude. When $V>e / 2 C$, charge transport is mainly determined by single-electron tunneling.

In the ideal case, for $e / C_{c}<V<e / 4 C$, the currents in both arrays are equal in magnitude and charge transport is determined by pure exciton tunneling. However, a number of effects give rise to deviations from this ideal behavior. In other words, a single-electron tunneling event in the biased array is not always directly followed by a tunneling event in the other array.

Even at zero temperature, there is a finite probability for higher-order cotunneling processes to occur, where in one event an electron is transferred through the voltage-biased array. This process does not contribute to charge transport in the other array. For the three-junction arrays, the rate of cotunneling through the entire array is proportional to $\left(R_{K} / R\right)^{3}$, while the exciton tunneling rate is proportional 
(a)

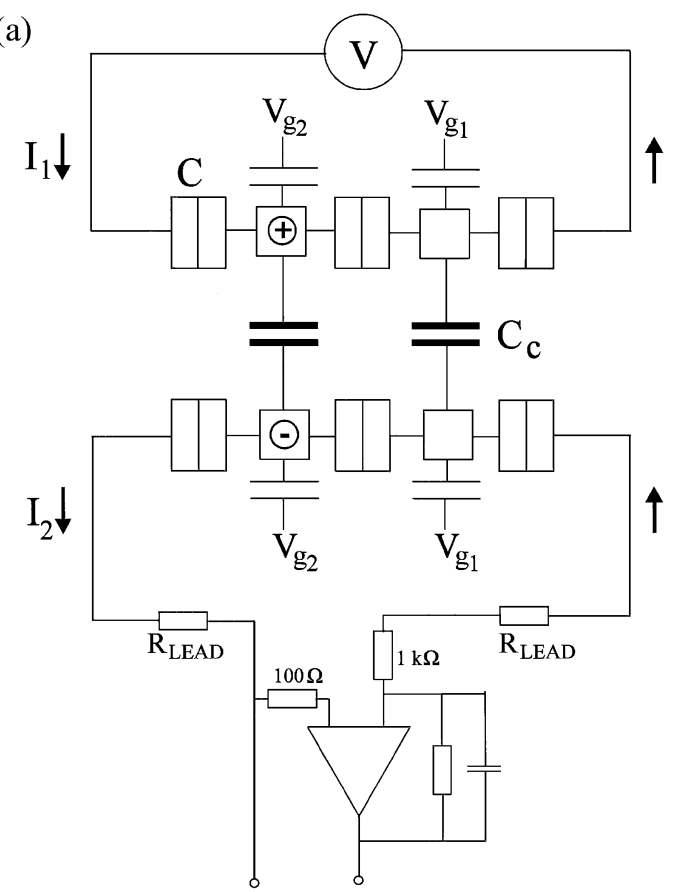

(b)

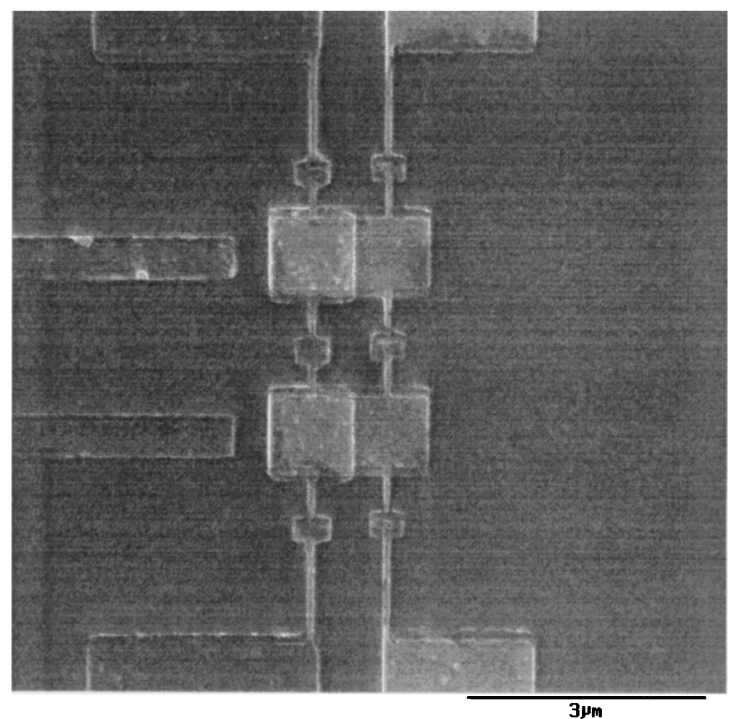

FIG. 1. (a) Schematic of the capacitively coupled 1D arrays. The upper array (array 1) is voltage biased, while the current is measured simultaneously in both arrays. The dc impedance seen by the bottom array (array 2) is mainly determined by the wiring and the filters in the dilution refrigerator $\left(R_{\text {lead }}\right)$ and is a few $\mathrm{k} \Omega$. In order to measure the current, the arrays were directly connected to an amplifier (used in a current-to-voltage converter circuit), as specifically indicated in the figure for the case of array 2 . The feedback resistor and capacitor of the amplifier have typical values of $100 \mathrm{M} \Omega$ and $0.1 \mathrm{nF}$. The offset of the amplifier was carefully adjusted to zero in order to eliminate any voltage difference between the inputs of the amplifier. The coupling capacitance $C_{c}$ is larger than the junction capacitance $C$. The gate voltages $V_{g 1}$ and $V_{g 2}$ can compensate for the random offset charges on the islands. Complete compensation is, however, not possible in this setup because there are only two gate voltages for four islands. The charge configuration of an electron-hole pair is schematically indicated. The electron-hole pair is created after an electron has tunneled into array 2 and out of array 1, thereby lowering the electrostatic energy of the system at small bias voltages. The electron-hole pair can move through the system, resulting in a current in both arrays. The currents are equal in magnitude but opposite in direction. (b) SEM photograph of the two capacitively coupled 1D arrays. The coupling capacitance is formed by the overlap capacitance of two islands belonging to different arrays. The area of the overlap is about $1 \mu \mathrm{m}^{2}$ and the dielectric consists of a few $\mathrm{nm}$ aluminum oxide and $10 \mathrm{~nm}$ silicon monoxide. Treating the overlap as a parallel-plate capacitor, the capacitance can be estimated to be approximately $5 \mathrm{fF}$. Each of the two gate electrodes, evaporated in the same step as the array in the bottom layer, has a comparable capacitance to two overlapping islands.

to $\left(R_{K} / R\right)^{2}$. Only for $R \gg R_{K}$, the contribution of the first process is negligible.

The above description of the exciton transport through the coupled arrays is valid for $C_{c} \gg C$. At smaller ratios of $C_{c} / C$, the difference between the electrostatic energies for excitons and single electrons in the array becomes smaller. At $C_{c} / C<5$, exciton transport can coexist only with single-electron tunneling. Pure exciton transport is not possible [10].

The electrostatic energy of a single electron on one of the islands of the arrays is influenced by the presence of a background charge close to this island [4]. The background charge can be an impurity charge in the substrate or in the oxides of tunnel junctions and overlap capacitors. If the background charges are not moving they can be compensated by a gate voltage that is capacitively coupled to the island. As shown in Fig. 1(a), each of the two gate voltages couples to a pair of islands of different arrays [see also Fig. 1(b)]. For technical reasons, it is hard to realize a separate gate electrode for each island without a considerable cross capacitance. The fact that we only have two gate voltages implies that we cannot fully compensate for four, possibly different, background charges. We can choose the gate voltages is such a way that the Coulomb blockade for single-electron tunneling is maximized in each array. This gives, however, no guarantee that the theoretical maximum of the Coulomb blockade is found. The theoretical maximum is reached in the absence of background charges. As a consequence, the electrostatic potentials of the island can be different from the optimum that enables pure exciton transport.

A finite temperature can make exciton transport possible at voltages lower than $e / C_{c}$. Exciton transport is possible through thermally activated cotunneling $[6,11,12]$. Moreover, single-electron tunneling can take place at voltages lower than the zero-temperature threshold $e / 2 C$. The single-electron tunneling disturbs the pure 
exciton transport. This results in a smaller ratio between the currents of the different arrays.

We have fabricated the capacitively coupled 1D arrays of Fig. 1(a) using a multilayer process [13]. First, one array was fabricated, together with the two gate electrodes, using standard electron beam lithography and shadowevaporation techniques. The two square islands of the array have an area of about $1 \mu \mathrm{m}^{2}$ each. In the next step, we evaporated a $10 \mathrm{~nm}$ layer of silicon monoxide to cover the array. In between these steps the sample has been taken out of the evaporation chamber and exposed to atmospheric pressure. In this way, an aluminum-oxide layer of a few $\mathrm{nm}$ is formed at the surface of the aluminum. This oxide layer is thicker than the oxide layer of the small $\mathrm{Al}-\mathrm{Al}_{2} \mathrm{O}_{3}-\mathrm{Al}$ tunnel junctions of the arrays. The junction oxide layer is formed in an oxygen environment at a controlled pressure of about 0.13 mbar. In the last step, we fabricated the second array close to the first one, in such a way that the islands of the two arrays were overlapping in pairs, over an area of about $1 \mu \mathrm{m}^{2}$. This overlap was realized by making the islands of the second array larger (about $2 \times 1 \mu \mathrm{m}^{2}$ ). A scanning electron microscopy (SEM) photograph of the sample is shown in Fig. 1(b).

We have made measurements on the capacitively coupled arrays in a dilution refrigerator with a base temperature of $10 \mathrm{mK}$. The leads to the sample we filtered with $R C$ filters and high-frequency copper powder filters at mixingchamber temperature. Although the dilution refrigerator was kept at base temperature during the measurements, the effective electron temperature can be higher (typically about $50 \mathrm{mK}$ in our setup [12]) due to self-heating, highfrequency radiation, and noise. We plot the $I-V$ characteristic of array 1, with current $I_{1}$, in Fig. 2. We observe

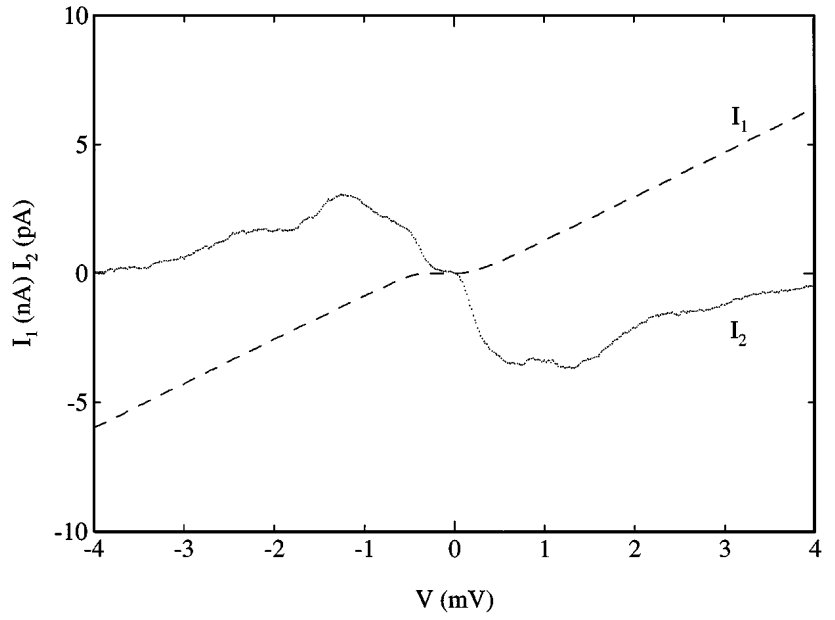

FIG. 2. Measured $I-V$ characteristics of the capacitively coupled 1D arrays, array 1 is voltage biased ( $V$ on the $x$ axis). The current is measured simultaneously in both arrays. Note the different current scales for $I_{1}$ (array 1, dashed line) and $I_{2}$ (array 2, small dots). a Coulomb blockade for single-electron tunneling with an estimated threshold voltage of $V_{\mathrm{SET}, 1} \approx 0.11 \mathrm{mV}$. From the $I-V$ characteristic at voltages much larger than the threshold voltage $V_{\mathrm{SET}, 1}$ we find that the total tunnel resistance $R_{\Sigma, 1}$ of the three junctions of array 1 is $574 \mathrm{k} \Omega$. If we voltage-bias only array 2 we find $V_{\mathrm{SET}, 2} \approx 0.10 \mathrm{mV}$ and $R_{\Sigma, 2}=371 \mathrm{k} \Omega$. Also in Fig. 2 we show the current in array 2 that was not biased. We clearly observe a current $\left(I_{2}\right)$ in the opposite direction. Note the different current scales for $I_{1}$ and $I_{2}$. In this voltage range, we see that the current $I_{2}$ is first increasing, reaches a maximum around $V=1 \mathrm{mV}$, and starts decreasing to reach zero at about $4 \mathrm{mV}$. The maximum in the current $I_{2}$ in Fig. 2 is likely to be reached when the average time between tunnel events in array $1, e / I_{1}$, becomes of the order of the reaction time of array 2, which is typically the $R C_{c}$ time. We also observe that $I_{2}$ is much smaller than $I_{1}$ for $V>V_{\mathrm{SET}, 1}$. At these voltages, the charge transport in array 1 is dominated by single-electron tunneling. There is only a small contribution to the current, coming from exciton tunneling.

In Fig. 3 we show a blowup of the $I-V$ characteristic of Fig. 2. We find, for $V<V_{\mathrm{SET}, 1} \approx 0.11 \mathrm{mV}$, that $I_{2}$ is of the same order of magnitude as $I_{1}$. At low voltages, we observe a rounded Coulomb blockade for exciton tunneling, due to thermal activation at finite temperatures.

From the size of the overlap capacitors, we estimate the coupling capacitance to be about $5 \mathrm{fF}$, giving $V_{\mathrm{exc}} \approx$ $0.03 \mathrm{mV}$. We can estimate the junction capacitances from the voltage $V_{\mathrm{SET}}=e / 2 C$, at which single-electron tunneling becomes the dominant transport mechanism and the current rises sharply. However, for this we assume

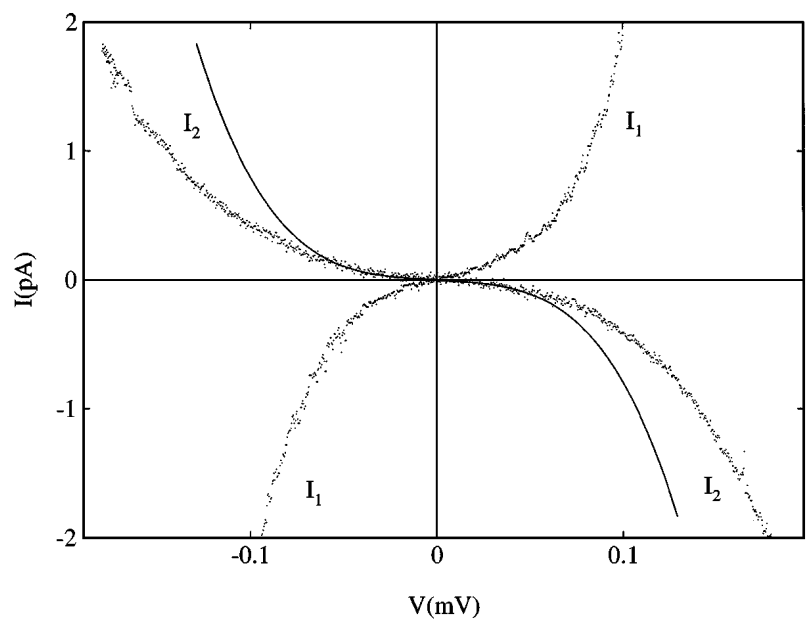

FIG. 3. Same plot as in Fig. 2, now for low bias voltages. Here, the current scales are the same for $I_{1}$ and $I_{2}$. The solid line is the theoretical prediction of the current carried by excitons, given by Eq. (2). The theoretical prediction is valid for voltages close to the threshold voltage for exciton tunneling, $V-V_{\mathrm{exc}} \ll V_{\mathrm{exc}}$ (if $V>0$ ). The parameters are $C=0.8 \mathrm{fF}, R=\sqrt{\frac{R_{\Sigma, 1}}{3} \frac{R_{\Sigma, 2}}{3}} \approx 150 \mathrm{k} \Omega, T=50 \mathrm{mK}$, and $C_{c}=$ $5 \mathrm{fF}\left(V_{\text {exc }}=0.03 \mathrm{mV}\right)$. 
that all junctions are equal and that all background charges are compensated. We find somewhat different values of $V_{\text {SET }}$ for array 1 and array 2 . We can give an estimate of $C$ to be $0.7 \mathrm{fF}$ for array 1 and $0.8 \mathrm{fF}$ for array 2 .

In Fig. 3, we also plot the theoretical prediction for the current for pure exciton transport close to the threshold value $V_{\text {exc }}[10]$ :

$$
I=\frac{\hbar C^{2}\left[V-V_{\mathrm{exc}}\right]}{\pi R^{2} e^{4}} \frac{\left[V-V_{\mathrm{exc}}\right]^{2}+\left[4 \pi k_{B} T / e\right]^{2}}{1-\exp \left[-e\left(V-V_{\mathrm{exc}}\right) / 2 k_{B} T\right]},
$$

where we take $R=\sqrt{\frac{R_{\Sigma, 1}}{3} \frac{R_{\Sigma, 2}}{3}} \approx 150 \mathrm{k} \Omega$ as the effective junction resistance. This equation is valid for $V-$ $V_{\text {exc }} \ll V_{\text {exc }}$ and low temperatures $k_{B} T \ll e^{2} / C_{c}$. We use $C=0.8 \mathrm{fF}$ and $C_{c}=5 \mathrm{fF}$. An effective temperature $T=50 \mathrm{mK}$ is introduced to account for self-heating and heating by external high-frequency radiation and noise. We observe that the current $I_{2}$, at voltages close to $V_{\text {exc }}$, is well described by Eq. (2). Nevertheless, we have to be careful not to make any quantitative statement, since we do not have an accurate knowledge of all parameters. Also, we find that the current in array 1 is somewhat larger than the current in array 2, even at the lowest voltages. We believe that this is caused by a small contribution to the current from single-electron tunneling, coexisting with exciton transport.

We have also investigated the electrical leakage of the overlap capacitors. We applied a voltage difference between array 1 and array 2 and we measured the current flowing through the junctions and the overlap capacitors. We applied voltage differences up to $5 \mathrm{mV}$, which is much larger than the Coulomb blockade voltage for single-electron tunneling. We found a current that is lower than our measuring sensitivity of about $0.05 \mathrm{pA}$. Although the islands of different arrays seem to be electrically well isolated, we cannot exclude the possibility that occasionally an electron moves through the coupling capacitors, thereby, for example, annihilating an exciton. The probability for this to happen is, however, negligible compared with other tunnel processes.

It is possible to numerically calculate the currents in both arrays by solving the master equation for the probabilities to find a certain number of excess electrons on the islands. All possible tunnel processes have to be included. However, since we cannot determine all parameters accurately, we have not performed a full quantitative comparison to theory.

A similar effect as described in this paper has been observed in coupled two-dimensional electron gases $[14,15]$. In that case, the driving and the induced current were in the same direction. The observed ratio of induced and driving current is much smaller than the one reported in this paper.

In conclusion, we have observed electron-hole transport in two capacitively coupled 1D arrays of small tunnel junctions. We find that the pure exciton transport through the system is disturbed by a number of effects. Nevertheless, we have demonstrated that a current can be induced in the array without a bias voltage. The induced current is comparable in magnitude with the current in the biased array and opposite in direction. This effect is a direct consequence of the Coulomb interaction of electrons.

We would like to acknowledge the Dutch Foundation for Fundamental Research on Matter (F. O. M.) for financial support. We would like to thank Yu. V. Nazarov and D. V. Averin for valuable discussions.

*Present and permanent address: Philips Research Laboratories, Prof. Holstlaan 4, 5656 AA Eindhoven, The Netherlands.

Electronic address: matters@natlab.research.philips.com

[1] L. J. Geerligs, V.F. Anderegg, P.A. M. Holweg, J.E. Mooij, H. Pothier, D. Estève, C. Urbina, and M. H. Devoret, Phys. Rev. Lett. 64, 2691 (1990).

[2] H. Pothier, P. Lafarge, P. F. Orfila, C. Urbina, D. Estève, and M.H. Devoret, Physica (Amsterdam) 169B, 573 (1991); H. Pothier, P. Lafarge, C. Urbina, D. Estéve, and M. H. Devoret, Europhys. Lett. 17, 249 (1992).

[3] P. Lafarge, H. Pothier, E. R. Williams, D. Estève, C. Urbina, and M. H. Devoret, Z. Phys. B 85, 327 (1991).

[4] D. V. Averin and K. K. Likharev, in Mesoscopic Phenomena in Solids, edited by B. L. Altshuler, P. A. Lee, and R. A. Webb (Elsevier, Amsterdam, 1991), p. 173.

[5] D. V. Averin and A. A. Odintsov, Phys. Lett. A 140, 251 (1989).

[6] D. V. Averin and Yu. V. Nazarov, Phys. Rev. Lett. 65, 2446 (1990).

[7] L. J. Geerligs, D. V. Averin, and J.E. Mooij, Phys. Rev. Lett. 65, 3037 (1990).

[8] H. Dalsgaard Jensen and J. M. Martinis, Phys. Rev. B 46, 13407 (1992).

[9] S. M. Verbrugh, Ph.D. thesis, Delft, 1995.

[10] D. V. Averin, A. N. Korotkov, and Yu. V. Nazarov, Phys. Rev. Lett. 66, 2818 (1991).

[11] T. M. Eiles, G. Zimmerli, H. D. Jensen, and J. M. Martinis, Phys. Rev. Lett. 69, 148 (1992).

[12] L. J. Geerligs, M. Matters, and J.E. Mooij, Physica (Amsterdam) 194B-196B, 1267 (1994).

[13] E. H. Visscher, S. M. Verbrugh, J. Lindeman, P. Hadley, and J. E. Mooij, Appl. Phys. Lett. 66, 305 (1995).

[14] P. M. Solomon, P. J. Price, D. J. Frank, and D. C. La Tulipe, Phys. Rev. Lett. 63, 2508 (1989).

[15] T. J. Gramila, J.P. Eisenstein, A.H. MacDonald, L. N. Pfeiffer, and K. W. West, Phys. Rev. Lett. 66, 1216 (1991). 